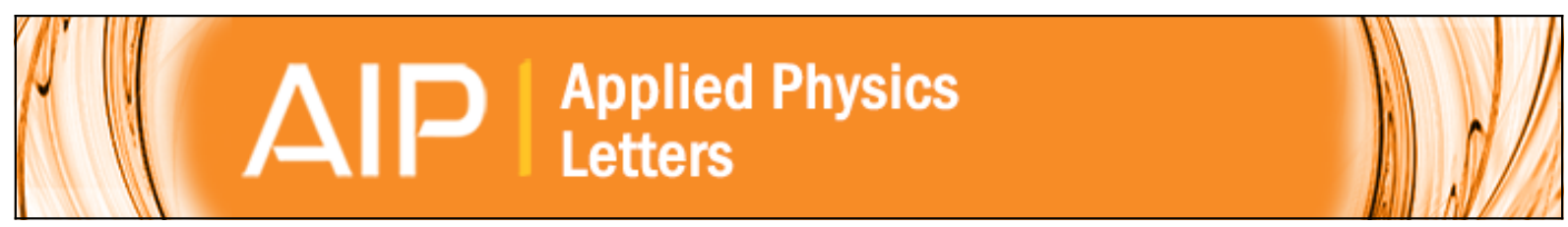

\title{
Stretchable nanocomposite electrodes with tunable mechanical properties by supersonic cluster beam implantation in elastomers
}

F. Borghi, C. Melis, C. Ghisleri, A. Podestà, L. Ravagnan, L. Colombo, and P. Milani

Citation: Applied Physics Letters 106, 121902 (2015); doi: 10.1063/1.4916350

View online: http://dx.doi.org/10.1063/1.4916350

View Table of Contents: http://scitation.aip.org/content/aip/journal/apl/106/12?ver=pdfcov

Published by the AIP Publishing

\section{Articles you may be interested in}

Mechanically stretchable and tunable metamaterial absorber

Appl. Phys. Lett. 106, 091907 (2015); 10.1063/1.4914502

Temperature-dependent electro-mechanical actuation sensitivity in stiffness-tunable BaTiO3/polydimethylsiloxane dielectric elastomer nanocomposites

Appl. Phys. Lett. 106, 092904 (2015); 10.1063/1.4914012

Inkjet-printed stretchable single-walled carbon nanotube electrodes with excellent mechanical properties

Appl. Phys. Lett. 104, 113103 (2014); 10.1063/1.4868633

Stretchable metal-elastomer nanovoids for tunable plasmons

Appl. Phys. Lett. 95, 154103 (2009); 10.1063/1.3247966

Dynamic Mechanical Properties of Fluorochemical Elastomers

Trans. Soc. Rheol. 2, 23 (1958); 10.1122/1.548822

Want to publish your paper in the \#1 MOST CITED journal in applied physics?

With Applied Physics Letters, you can.

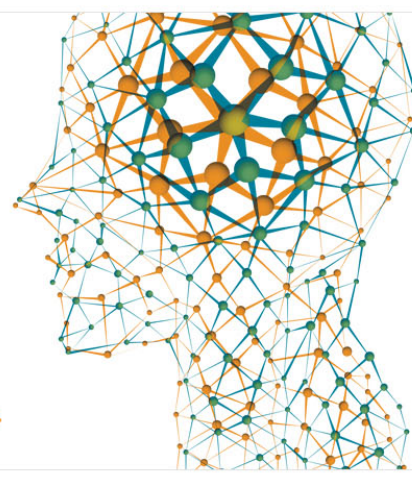




\title{
Stretchable nanocomposite electrodes with tunable mechanical properties by supersonic cluster beam implantation in elastomers
}

\author{
F. Borghi, ${ }^{1}$ C. Melis, ${ }^{2}$ C. Ghisleri, ${ }^{3}$ A. Podestà, ${ }^{1}$ L. Ravagnan,${ }^{3}$ L. Colombo, ${ }^{2}$ and P. Milani $\left.{ }^{1}, a\right)$ \\ ${ }^{1}$ CIMAINA and Dipartimento di Fisica, Università degli Studi di Milano, via Celoria 16, 20133 Milano, Italy \\ ${ }^{2}$ Dipartimento di Fisica, Università di Cagliari, Cittadella Universitaria, I-09042 Monserrato (Ca), Italy \\ ${ }^{3}$ WISE srl, Piazza Duse 2, 20122 Milano, Italy
}

(Received 23 February 2015; accepted 17 March 2015; published online 25 March 2015)

\begin{abstract}
We demonstrate the fabrication of gold-polydimethylsiloxane nanocomposite electrodes, by supersonic cluster beam implantation, with tunable Young's modulus depending solely on the amount of metal clusters implanted in the elastomeric matrix. We show both experimentally and by atomistic simulations that the mechanical properties of the nanocomposite can be maintained close to that of the bare elastomer for significant metal volume concentrations. Moreover, the elastic properties of the nanocomposite, as experimentally characterized by nanoindentation and modeled with molecular dynamics simulations, are also well described by the Guth-Gold classical model for nanoparticle-filled rubbers, which depends on the presence, concentration, and aspect ratio of metal nanoparticles, and not on the physical and chemical modification of the polymeric matrix due to the embedding process. The elastic properties of the nanocomposite can therefore be determined and engineered a priori, by controlling only the nanoparticle concentration. (C) 2015 AIP Publishing LLC. [http://dx.doi.org/10.1063/1.4916350]
\end{abstract}

Dielectric elastomer actuators (DEAs) are attracting a rapidly increasing interest as lightweight and inexpensive electromechanical transducers for the fabrication of soft robots, ${ }^{1,2}$ smart actuators, ${ }^{3}$ haptic interfaces, and energy harvesting systems. ${ }^{4,5}$ DEAs are based on an elastomer sandwiched between two electrodes: this configuration results in a deformation (in plane or out of plane) under compression when the two electrodes are electrically polarized using voltages of several $\mathrm{kV}$. $^{2,4}$

In view of a widespread utilization of DEAs and of their integration in microdevices, one of the major challenges is the (micro)fabrication of compliant and well-adherent electrodes able to sustain a very large number of deformations (millions of cycles) while remaining electrically conductive. Their mechanical properties must be, as much as possible, similar to those of the elastomeric material in order not to alter the stiffness and deformation characteristics of the actuator. ${ }^{4}$ Electrodes made by metal thin films deposited by sputtering or evaporation show poor adhesion, deterioration, and delamination after few deformation cycles. ${ }^{6,7}$ Moreover, the Young's modulus of a metal film is several orders of magnitude higher than that of dielectric elastomers (50-100 GPa compared to $0.2-1 \mathrm{MPa})$, causing a very low actuation strain. ${ }^{4}$

Low-energy implantation of metallic ions in elastomers has been used for the fabrication of thin polymer-metal nanocomposite layers acting as electrodes in DEAs. ${ }^{89}$ The nanocomposite layer is formed by metallic clusters, resulting from the aggregation of atomic species subsequent to ion implantation, embedded in the elastomeric matrix. This technique assures a good adhesion to the bare elastomer and a high degree of compliance. ${ }^{8,9}$

\footnotetext{
${ }^{\text {a) }}$ Author to whom correspondence should be addressed. Electronic mail: pmilani@mi.infn.it.
}

Recently, we showed that neutral metal clusters, accelerated in a supersonic expansion, can be implanted in elastomers to form an electrically conductive nanocomposite..$^{10,11}$ This process, called supersonic cluster beam implantation (SCBI), avoids both sample heating and charging and it is fully compatible with stencil mask micropatterning and liftoff technology. ${ }^{11,12}$ Stretchable electrodes obtained by $\mathrm{Au}$ nanoparticles (NPs) implantation in polydimethylsiloxane (PDMS) are able to withstand more than $1 \times 10^{6}$ of uniaxial stretching cycles (at $40 \%$ strain) preserving finite and reproducible electrical resistance. ${ }^{10}$

Here, we demonstrate the fabrication of electrodes based on $\mathrm{Au} / \mathrm{PDMS}$ nanocomposite with a Young's modulus depending solely on the amount of metal clusters implanted in the elastomeric matrix. We show both experimentally and by numerical simulations that the mechanical properties of the nanocomposite can be maintained close to that of the bare elastomer for significant metal volume concentrations. The elastic properties of the Au-PDMS nanocomposites are experimentally characterized by nanoindentation and modeled with molecular dynamics (MD) simulations and the Guth-Gold classical model. ${ }^{13-15}$

We fabricated Au-PDMS electrodes by implanting different quantities of neutral Au nanoparticles with a size distribution reported in Fig. 1(a) (as obtained by transmission electron microscopy (TEM) images. We used a deposition apparatus equipped with a Pulsed Microplasma Cluster Source (PMCS), as described in Ref. 10. Briefly, a PMCS consists a ceramic body with a cavity in which a solid $\mathrm{Au}$ target (purity $99.9 \%$ ) is vaporized by a localized electrical discharge ignited during the injection of a pulse of inert gas (He or Ar) at high pressure (40 bars). The metal atoms, sputtered from the target, aggregate in the source cavity to form metal clusters; the mixture of clusters and inert gas expands subsequently through a nozzle forming a supersonic beam 

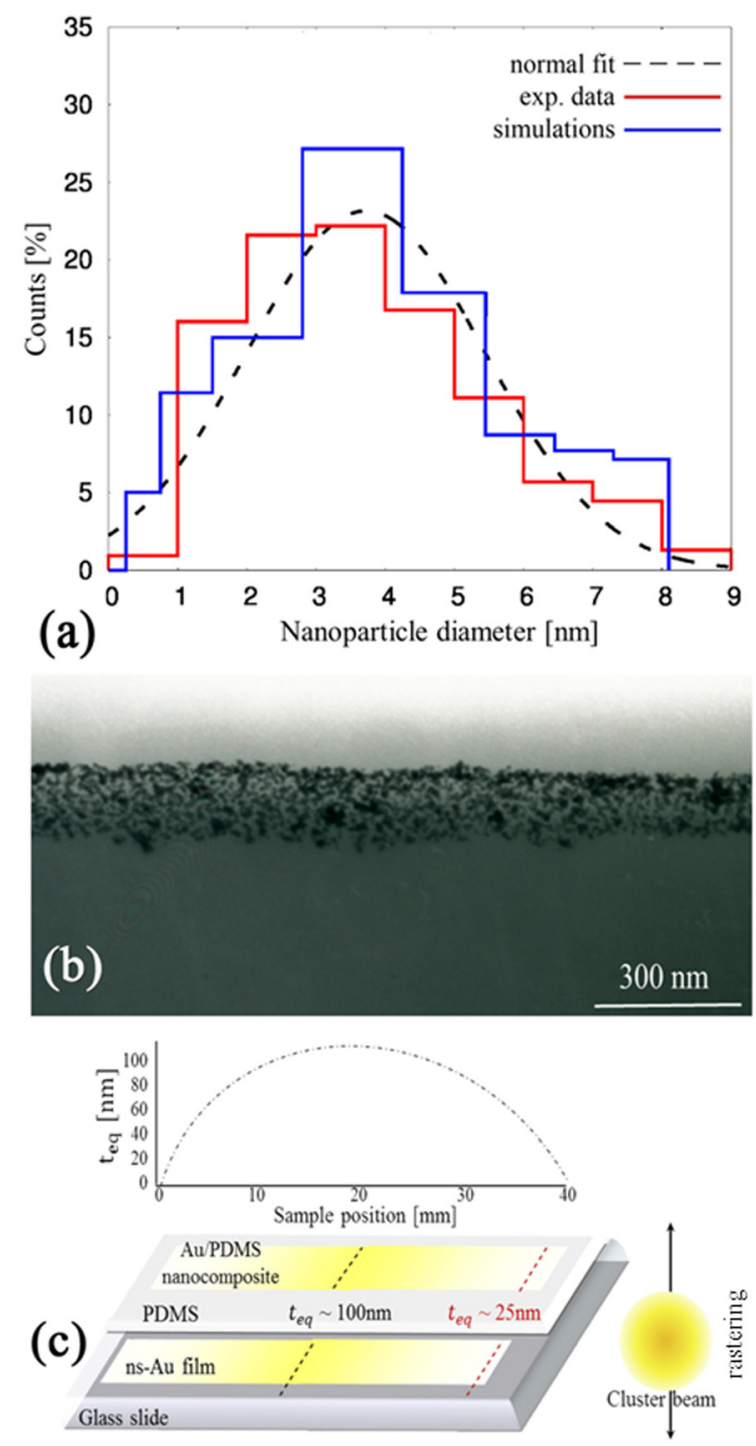

FIG. 1. (a) Experimental (red) and simulated (blue) Au cluster diameter distribution. The mean value of the normal distribution is $3.7 \pm 1.7 \mathrm{~nm}$. (b) TEM image of a thin section of the PDMS implanted with an equivalent thickness of $30 \mathrm{~nm}$. The penetration depth of the nanoparticles is approximately $180 \mathrm{~nm}$. (c) Schematic representation of a typical sample. A symmetric gradient of $\mathrm{Au}$ NPs on the clean glass surface is used to measure the equivalent thickness $t_{\mathrm{eq}}$ of implanted $\mathrm{Au}$ (which ranges from $100 \mathrm{~nm}$ in the center to $25 \mathrm{~nm}$ at the border). The same gradient of Au NPs implanted on a uniform PDMS film, covering the upper half of the glass slide, is used to characterize the Young's modulus for different equivalent thicknesses. A portion of PDMS is left unimplanted as reference in the nanomechanical analysis.

into an expansion chamber kept at $10^{-6}$ mbars. Electrically neutral nanoparticles exiting the PMCS are aerodynamically accelerated in a highly collimated beam with divergence lower than $1^{\circ}$ and with a kinetic energy typically of $0.5 \mathrm{eV} /$ atom. ${ }^{16}$ The central part of the supersonic cluster beam enters, through a skimmer, a second vacuum chamber (deposition chamber) where the beam is intercepted by the PDMS substrate. The implanted Au nanoparticles volume fraction (Au volume concentration) is defined as the ratio between the total volume of the metal nanoparticles (the metal filler) and the volume of polymer in which the nanoparticles are implanted. Considering a homogeneously filled nanocomposite, this corresponds to the ratio between the equivalent thickness of the implanted nanoparticles and the thickness of the nanocomposite layer. ${ }^{10}$ The thickness of the nanocomposite layer (nanoparticle implantation depth) can be obtained by TEM characterization (Fig. 1(b)). The equivalent thickness $t_{\text {eq }}$ of nanoparticles implanted into the PDMS is obtained by measuring, by Atomic Force Microscopy (Bioscope Catalyst AFM, Bruker), the thickness of the Au cluster-assembled film deposited on a bare glass substrate region next to the PDMS film (Fig. 1(c)).

In order to systematically characterize the role of the nanoparticle volume fraction on the nanocomposite mechanical properties, we produced samples with a nanoparticle density gradient, as schematically shown in Fig. 1(c). This is obtained by rastering the PDMS substrate against the supersonic cluster beam only along one axis in order to obtain a homogeneous nanocomposite in the direction parallel to the raster, with a gradient in the amount of implanted nanoparticles along the orthogonal axis. The same nanoparticle gradient is deposited on the glass substrate to allow the direct measurement of the equivalent thickness. PDMS and glass substrates underwent an implantation and deposition process, respectively, for $27 \mathrm{~min}$ with a deposition rate of $0.06 \mathrm{~nm}$ $\mathrm{s}^{-1}$, reaching $\mathrm{t}_{\mathrm{eq}}=100 \mathrm{~nm}$ in the center of the sample.

PDMS substrates were produced with a Sylgard 184 Elastomer Kit by mixing the base and the curing agent in a 10:1 ratio for about $15 \mathrm{~min}$.

The evolution of the Young's modulus of Au-PDMS nanocomposites when $t_{\mathrm{eq}}$ is varying has been characterized by recording force vs indentation curves ${ }^{17,18}$ with an AFM equipped with custom-made micro-probes consisting of spherical silica microparticles attached to tipless cantilevers ${ }^{19}$ with force constant $\mathrm{k}=2.9 \mathrm{~N} / \mathrm{m}$. The AFM probe is periodically pushed against the nanocomposite surface and the elastic indentation of the material is measured as a function of the total applied force (Fig. 2). The use of spherical probes with a diameter of $2 \mu \mathrm{m}$ allows well-defined contact geometry (sphere on flat) and the use of analytical contact

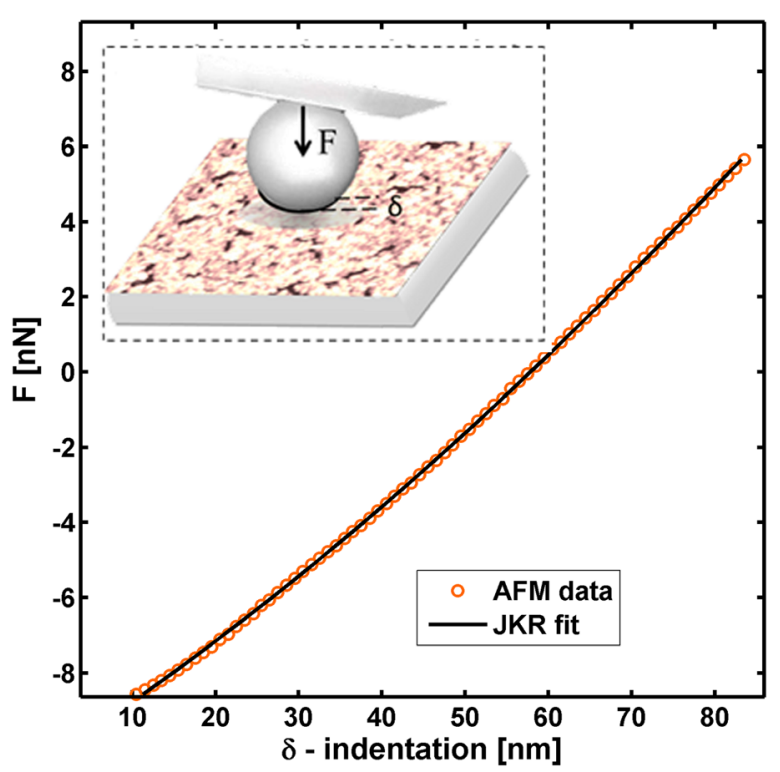

FIG. 2. A representative force-indentation curve acquired on the Auimplanted PDMS sample, with the modified JKR fit superimposed to the experimental data. In the inset, a schematic representation of the AFM indentation test is shown. 
mechanics models for data fitting; moreover, large spherical probes provide an averaged and stable mechanical readout, representative of the mesoscopic properties of the nanocomposite material. ${ }^{19,20}$

Twelve different regions of the Au-PDMS nanocomposite sample have been tested: 100 force curves have been typically acquired in three different positions (separated by $10 \mu \mathrm{m}$ ) of each region. For large, adhesive contacts and soft surfaces, the Johnson-Kendall-Roberts (JKR) model is appropriate to describe indentation; ${ }^{21,22}$ we have used a modified JKR equation to fit data, which takes into account also a constant capillary adhesive force due to the presence of a water meniscus (measurements have been carried out in ambient conditions, at relative humidity of 35\%) (Fig. 2). The Poisson coefficient has been set to 0.5 , as typical for polymers. ${ }^{17,23}$ From force-indentation curves, the effective Young's modulus of the composite structure formed by the thick PDMS substrate and the thin nanocomposite AuPDMS layer is extracted. ${ }^{9}$ The values of the effective Young's modulus have been normalized by the value of the modulus of the unimplanted PDMS, in order to better evaluate the $\mathrm{Au}$ concentration-dependent variations and compare to numerical simulation data (the same normalization has been applied to in silico data).

The characterization of the nanocomposite Young's modulus via AFM indentation necessarily implies the use of mesoscopic models aimed at the description of the tip-sample interactions. The transferability of our findings to a macroscopic "bulk" scale is not straightforward; on the other hand, the typical dimensions of our nanocomposite electrodes do not allow the standard elasticity characterization approach for bulk samples based on traction-elongation cycles. ${ }^{24}$ Hence, we decided to complement the experimental investigation by all-atom calculations aimed at predicting the dependence of the Young's modulus upon Au content by simulated tractionelongation cycles on nanocomposite samples.

Computer-generated nanocomposite samples have been obtained by simulating multiple implantations of Au clusters into the PDMS substrate. The implantation process strictly followed the procedure described in Refs. 11 and 25, although in the present case more than one clusters were implanted, with the size distribution obtained by TEM images reported in Fig. 1(a). Overall five different Au-PDMS nanocomposite samples were generated with $\mathrm{Au}$ volume concentration ranging between $8 \%$ and $29 \%$. The corresponding number of particles in our simulation cells ranged between 350000 and 450000 . An increasing number of Au nanoparticles were sequentially implanted on the PDMS substrate. For computational convenience in computer-generating the samples, the implantation was emulated by setting an impact energy of $2 \mathrm{eV} /$ atom and a deposition rate of $50 \mathrm{ps}^{-1}$. Such a rate, although higher than in typical SCBI experiments, was nevertheless small enough to allow the full relaxation of the PDMS substrate upon each implantation event. Therefore, like in real SCBI experiments, the next cluster impacted on a fully relaxed substrate. Interatomic interactions have been modeled as reported in Refs. 11 and 25.

Once the nanocomposite samples at different $\mathrm{Au}$ nanoparticle concentrations were generated, we estimated the corresponding Young's modulus by simulating traction- elongation cycles. In detail, each cubic simulation cell with edge $\mathrm{L}_{0}$ (containing an Au-PDMS sample with given $\mathrm{Au}$ content) was gently (strain rate $=1.75 \mathrm{~ns}^{-1}$ ) elongated at constant room temperature for $0.8 \mathrm{~ns}$, by imposing volume conservation. ${ }^{26}$ During the traction cycle, intermediate configurations have been saved every $0.2 \mathrm{~ns}$, providing four different strained samples (with same Au content). Their final extension $\mathrm{L}$ is defined as: $\mathrm{L}=\lambda \mathrm{L}_{0}$, where $\lambda=1.1,1.2,1.3$, and 1.4. At this stage, each strained sample was carefully relaxed at room temperature by a constant-volume annealing as long as $2.5 \mathrm{~ns}$, driving the corresponding atomic structure at its minimum-energy configuration, where the tensile stress $\sigma_{\mathrm{T}}$ was eventually calculated (such a stress value was calculated using the standard virial expression for the atomicscale stress tensor). ${ }^{27}$

A typical stress-strain plot from MD simulations is shown in Fig. 3 for an Au-PDMS composite containing 8\% of metallic clusters, providing evidence of the stress-strain dependence formulated in terms of the deformation parameters $\lambda^{2}-1 / \lambda$.

This dependence, which is as well found in all systems here investigated, stands for the robustness of the present results since it is in agreement with the prediction of elementary viscoelasticity theory, ${ }^{26}$ where it is proved that

$$
\sigma_{\mathrm{T}}=\mathrm{G}\left(\lambda^{2}-1 / \lambda\right) \text {. }
$$

In this framework, $\mathrm{G}$ is the elastic modulus of the elastomer, which is related to the corresponding Young's modulus through a very simple relation: $\mathrm{E}=3 \mathrm{G}$. The calculation of the stress-strain curve through MD simulations of a tractionelongation cycle directly offers a prediction on the Young's modulus of Au-PDMS composites.

Fig. 4 shows the normalized Young's modulus measured in silico and the normalized effective Young's modulus measured by AFM as a function of Au volume concentration. The modulus is basically constant (slightly decreasing in the case of experimental values) for concentration $\rho$ below $\sim 20 \%$; above this threshold, we observe an exponential increase of $\mathrm{E}$ up to 5 times $\mathrm{E}_{0}$ for $\rho \sim 30 \%$. The agreement between the experimentally determined effective modulus and the numerically determined modulus is remarkable. Both experimental and numerical data can be fitted by an analytical function in the form $1+\exp (-\mathrm{A}(\mathrm{B}-\mathrm{x})$ ) (the blue and red curves in Fig. 4), with $\mathrm{A}=0.3,0.2$ and $\mathrm{B}=23.1$, 24.3 for the experimental and numerical data, accordingly.

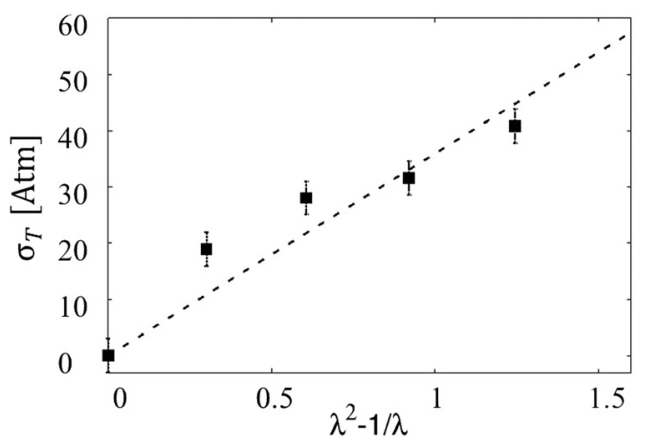

FIG. 3. $\sigma_{\mathrm{T}}$ vs. $\lambda^{2}-1 / \lambda$ for an Au-PDMS nanocomposite having $\mathrm{Au}$ volume concentration $\rho=8 \%$. The black line represents the linear fit obtained according to equation $\sigma_{\mathrm{T}}=\mathrm{G}\left(\lambda^{2}-1 / \lambda\right)$. 


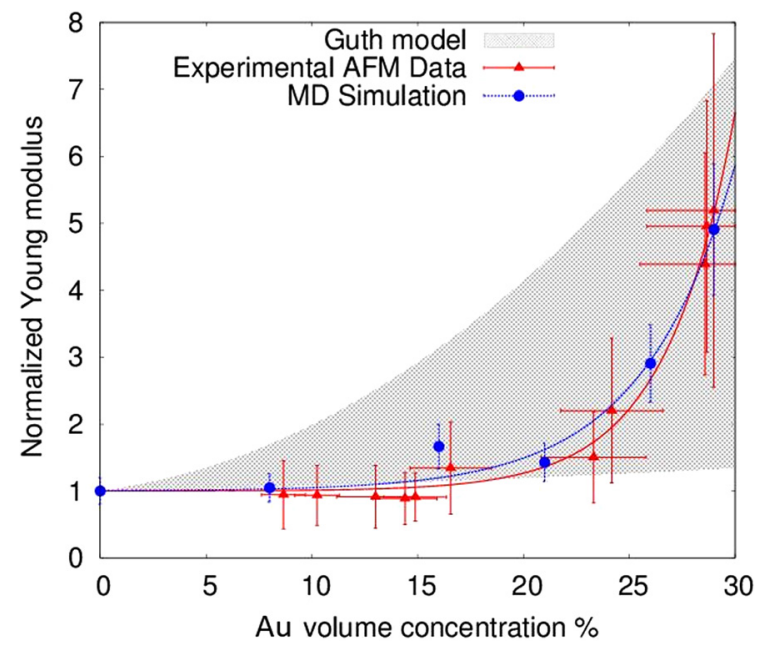

FIG. 4. Normalized $\mathrm{E} / \mathrm{E}_{0}$ vs. Au volume concentration $\rho$ calculated from dynamic mechanical simulations (blue dots) and nano-indentation AFM experiments (red triangles). The shaded region represents the prediction of the Guth-Gold model for different nanoparticles aspect ratios $(\alpha=1$ for the lower bound, $\alpha=6$ for the upper bound), calculated as E/ $\mathrm{E}_{0}=1+0.67(\alpha \rho)+1.62(\alpha \rho)^{2}$. The blue and red fitting curves are obtained by considering an analytical fitting function $1+\exp (-\mathrm{A}(\mathrm{B}-\mathrm{x}))$.

In the case of pristine PDMS, we estimated through the above traction-elongation MD protocol a Young's modulus $\mathrm{E}_{0}=6.4 \pm 0.8 \mathrm{MPa}$. This value is about 3 times larger than those measured in nanoindentation AFM experiments. This discrepancy is likely due to a different distribution of the length of the polymer chains: while in MD simulations this distribution is by construction unimodal (corresponding to a typical length of 40 monomers per chain), the corresponding distribution of real PDMS samples is not known in detail. We also remark that it has been shown ${ }^{28}$ that the Young's modulus in pristine PDMS can vary over 2 orders of magnitudes by increasing the chain length from 1 to 1000 . In order to reconcile simulations to experiments, the calculated/measured values of $\mathrm{E}$ in Au-PDMS composites have been renormalized to the corresponding value in pristine PDMS. This procedure, while straightforward, is very effective in separating the role of the implanted Au nanoclusters on the elastic properties of pristine PDMS (which is indeed the main target of this investigation) from the possible effects due to a different distribution of polymer chains.

It is remarkable that the agreement between the presented experimental/simulated results and the theoretical expectations of the classical Guth-Gold model ${ }^{13-15}$ has been proposed to describe the effect of a filler on the elastic properties of a continuous rubber matrix, as indicated by the shadowed area in Fig. 4. The Guth-Gold model has been proposed to predict the modification of the normalized Young's modulus $\mathrm{E} / \mathrm{E}_{0}$ of a rubber-like polymer filled with metal nanoparticles with a specific aspect ratio $\alpha$ and at a given volume fraction $\rho$, according to the following equation: ${ }^{15}$

$$
\mathrm{E} / \mathrm{E}_{0}=1+0.67(\alpha \rho)+1.62(\alpha \rho)^{2} .
$$

The shadowed region in Fig. 4 corresponds to the area spanned by the Guth-Gold curves obtained for different nanoparticle aspect ratios $\alpha$ ranging from 1 (lower bound, spherical particles) to 6 (upper bound, elongated particles).
The agreement is remarkable since the characterization approaches of the Young's modulus are based on very different assumptions, approximations, and length scales. The Guth-Gold model assumes that the modification of the nanocomposite elasticity only depends on the presence, concentration, and aspect ratio of the metal nanoparticles and not on the physical and chemical modification of the polymeric matrix due to the embedding process. This supports the assumption $^{10,11}$ that SCBI is not causing bond breaking events along the polymer chain, without modification of the underlying chemistry. Rather, only a microstructure evolution of the polymeric host is observed upon cluster incorporation. This, however, strongly affects the elastic properties of the polymeric matrix, as stated in Ref. 29. The comparison among different approaches reported in Fig. 4 actually indicates that SCBI is effectively a cold implantation technique, not damaging the chemical composition and structure of the polymeric chains and thus maintaining the mechanical properties of the polymeric substrate unaltered. Another interesting observation is that AFM indentation results shift toward a higher particles aspect ratio for increasing concentration of nanoparticles; this suggests that nanoparticles coalesce to form irregular larger aggregates towards the onset of a percolative structure, as already observed by electrical characterization of the nanocomposites. ${ }^{10}$

In conclusion, we have demonstrated both experimentally and theoretically the production of Au-PDMS nanocomposites with a Young's modulus depending solely on the amount of nanoparticle embedded in the elastomer. The elastic properties of the nanocomposite can be determined and engineered a priori, by controlling only one parameter thus allowing the preparation of DEAs electrodes with suitable mechanical properties.

A very important point is that the results of AFM indentation tests of the nanocomposite can be modeled and reproduced both by atomic-scale MD simulations and by the macroscopic Guth-Gold model: this shows that the assumptions of our approach are robust and they can be used to predict and design the functional and structural properties on a novel class of nanocomposites obtained by SCBI and used for stretchable electronics and optics. ${ }^{30}$

This work has been supported by project "ELDABI Elettronica Deformabile per Applicazioni Biomediche" (Project No. 26599138). We thank R. Simonetta for support in nanoindentation experiments.

\footnotetext{
${ }^{1}$ S. Bauer, S. Bauer-Gogonea, I. Graz, M. Kaltenbrunner, C. Keplinger, and R. Schwödiauer, Adv. Mater. 26, 149 (2014).

${ }^{2}$ I. A. Anderson, T. A. Gisby, T. G. McKay, B. M. O’Brien, and E. P. Calius, J. Appl. Phys. 112, 041101 (2012).

${ }^{3}$ A. P. Gerratt, B. Balakrisnan, I. Penskiy, and S. Bergbreiter, Smart Mater. Struct. 23, 055004 (2014).

${ }^{4}$ S. Rosset and H. R. Shea, Appl. Phys. A 110, 281 (2013).

${ }^{5}$ G. Kang, K. S. Kim, and S. Kim, Rev. Sci. Instrum. 82, 046101 (2011).

${ }^{6}$ O. Graudejus, B. Morrison, C. Goletiani, Z. Yu, and S. Wagner, Adv. Funct. Mater. 22, 640 (2012).

${ }^{7}$ C. L. Tuinea-Bobe, P. Lemoine, M. U. Manzoor, M. Tweedie, R. A. D'Sa, C. Gehin, and E. Wallace, J. Micromech. Microeng. 21, 115010 (2011).

${ }^{8}$ S. Rosset, M. Niklaus, P. Dubois, and H. R. Shea, Adv. Funct. Mater. 19, 470 (2009).

${ }^{9}$ M. Niklaus and H. R. Shea, Acta Mater. 59, 830-840 (2011).
} 
${ }^{10}$ G. Corbelli, C. Ghisleri, M. Marelli, P. Milani, and L. Ravagnan, Adv. Mater. 23, 4504 (2011).

${ }^{11}$ C. Ghisleri, F. Borghi, L. Ravagnan, A. Podestà, C. Melis, L. Colombo, and P. Milani, J. Phys. D: Appl. Phys. 47, 015301 (2014).

${ }^{12}$ M. Marelli, G. Divitini, C. Collini, L. Ravagnan, G. Corbelli, C. Ghisleri, A. Gianfelice, C. Lenardi, P. Milani, and L. Lorenzelli, J. Micromech. Microeng. 21, 045013 (2011).

${ }^{13}$ E. Guth and O. Gold, Phys. Rev. 53, 322 (1938).

${ }^{14}$ E. Guth, J. Appl. Phys. 16, 20 (1945).

${ }^{15}$ Y. P. Wu, Q. X. Jia, D. S. Yu, and L. Q. Zhang, Polym. Test. 23, 903 (2004).

${ }^{16}$ P. Piseri, H. V. Tafreshi, and P. Milani, Curr. Opin. Solid State Mater. Sci. 8, 195 (2004).

${ }^{17}$ E. K. Dimitriadis, F. Horkay, J. Maresca, B. Kachar, and R. S. Chadwick, Biophys. J. 82, 2798 (2002).

${ }^{18}$ H. J. Butt, B. Cappella, and M. Kappl, Surf. Sci. Rep. 59, 1 (2005).

${ }^{19}$ M. Indrieri, A. Podestà, G. Bongiorno, D. Marchesi, and P. Milani, Rev. Sci. Instrum. 82, 023708 (2011).
${ }^{20}$ L. Puricelli, M. Galluzzi, C. Schulte, A. Podestà, and P. Milani, "Nanomechanical and topographical imaging of living cells by Atomic Force Microscopy with colloidal probes," Rev. Sci. Instrum. (in press).

${ }^{21}$ K. L. Johnson, K. Kendall, and A. D. Roberts, Proc. R. Soc. London, Ser. A 324, 301 (1971).

${ }^{22}$ D. Tabor, J. Colloid Interface Sci. 58, 2 (1977).

${ }^{23}$ J. E. Mark, Polymer Data Handbook (Oxford University Press, New York, 1999).

${ }^{24}$ S. Lampman, Characterization and Failure Analysis of Plastics (ASM International Editor, 2003).

${ }^{25}$ R. Cardia, C. Melis, and L. Colombo, J. Appl. Phys. 113, 224307 (2013).

${ }^{26}$ M. Rubistein and R. H. Colby, Polymer Physics (Oxford University Press, 2003).

${ }^{27}$ S. Giordano, A. Mattoni, and L. Colombo, Rev. Comput. Chem. 27, 1 (2011).

${ }^{28}$ D. R. Heine, G. S. Grest, C. D. Lorenz, M. Tsige, and M. J. Stevens, Macromolecules 37, 3857 (2004).

${ }^{29}$ P. J. Flory, Polym. J. 17, 1 (1985).

${ }^{30}$ C. Ghisleri, M. A. C. Potenza, L. Ravagnan, A. Bellacicca, and P. Milani, Appl. Phys. Lett. 104, 061910 (2014). 